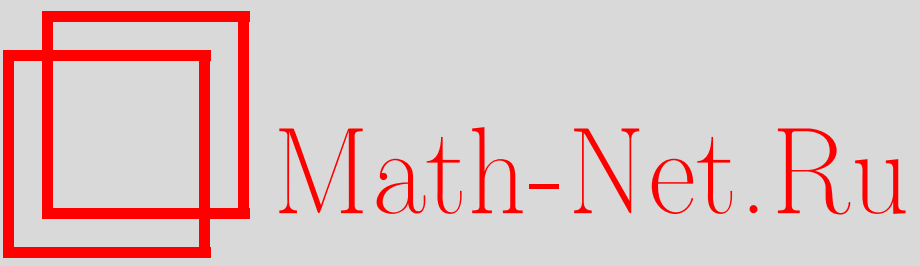

В. Э. Адлер, В. Г. Марихин, А. Б. Шабат, Лагранжевы цепочки и канонические преобразования Беклунда, ТМФ, 2001, том 129, номер 2, 163-183

DOI: https://doi.org/10.4213/tmf528

Использование Общероссийского математического портала Math-Net.Ru подразумевает, что вы прочитали и согласны с пользовательским соглашением

http://www.mathnet.ru/rus/agreement

Параметры загрузки:

IP : 107.22 .136 .117

26 апреля 2023 г., 16:49:07 
ТЕОРЕТИЧЕСКАЯ

И МАТЕМАТИЧЕСКАЯ

ФИЗИКА

Том 129, № 2

ноябрь, 2001

(C) 2001 г.

В.Э. Адлер* , В.Г. Марихин ${ }^{\dagger}$, А.Б. Шабат ${ }^{\dagger}$

\section{ЛАГРАНЖЕВЫ ЦЕПОЧКИ И КАНОНИЧЕСКИЕ ПРЕОБРАЗОВАНИЯ БЕКЛУНДА}

Рассматриваются преобразования Дарбу для операторов произвольного порядка, строится общая теория преобразований Беклунда на основе лагранжева формализма. Одевающая цепочка для уравнения Буссинеска и ее редукции являются показательными примерами к развиваемой общей теории. Обсуждаются также известные преобразования Беклунда для классических солитонных уравнений.

\section{1. ВВЕДЕНИЕ}

Одним из наиболее надежных методов анализа интегрируемости динамических систем является построение преобразований Беклунда (ПБ), т.е. преобразований, позволяюших получать новые решения данной динамической системы. Рассматривая композицию ПБ, можно построить дифференциально-разностные системы - интегрируемые цепочки, алгебраически эквивалентные рассматриваемым динамическим системам. Если данная динамическая система является гамильтоновой, то ПБ есть канонические преобразования, сохраняющие гамильтониан (точнее, разность гамильтонианов до и после преобразования Беклунда является полной производной).

Рассматриваемые нами цепочки имеют лагранжианы обшего вида

$$
\mathcal{L}=\int d x \sum F\left(q_{n}, q_{n+1}, q_{n, x}, q_{n+1, x}\right)
$$

Если лагранжиан $F=F\left(q, q_{1}, q_{x}, q_{1, x}\right)$ нелинеен по производным, то мы получаем одевающие цепочки для уравнений типа Буссинеска при $F_{q_{x}, q_{1, x}} \neq 0$ и цепочки типа Тоды и релятивистской цепочки Тоды, связанных с уравнениями типа нелинейного уравнения Шредингера (НУШ), при $F_{q_{x}, q_{1, x}}=0$. Для линейных по производным лагранжианов

$$
F=a\left(q_{n}, q_{n+1}\right) q_{n, x}+b\left(q_{n}, q_{n+1}\right)
$$

\footnotetext{
* Институт математики Уфимского научного центра РАН, Уфа, Россия. E-mail: adler@imat.rb.ru

${ }^{\dagger}$ Институт теоретической физики им. Л. Д. Ландау РАН, Москва, Россия.

E-mail: mvg@itp.ac.ru; shabat@itp.ac.ru
} 
особый интерес представляет случай, когда уравнения Эйлера-Лагранжа сводятся к цепочкам вида

$$
g\left(q_{n}, q_{n+1}, q_{n, x}, q_{n+1, x}\right)=0 .
$$

Этот случай охватьвает одеваюшие цепочки для уравнений типа Кортевега - де Фриза $(\mathrm{Kд} \Phi)$, синус-Гордон и соответствует гамильтоновым уравнениям

$$
J q_{t}=\frac{\delta H}{\delta q}
$$

с гамильтоновыми операторами $J=D_{x}, J=q_{x}^{-2} D_{x}+D_{x} q_{x}^{-2}$. В конце статьи рассматриваются одевающие цепочки для гамильтоновых уравнений типа Савады-Котера с гамильтоновыми операторами $J$ третьего порядка. Эти цепочки возникают как хорошо определенные редукции цепочки с лагранжианом

$$
F=q_{x}^{2}+2 q_{x} q_{1, x}+\left(q_{x}+q_{1, x}\right)\left(q_{1}-q\right)^{2}+\left(q_{1}-q\right)^{4}+\mu\left(q_{1}-q\right) .
$$

Последний и является лагранжианом одевающей цепочки для уравнения Буссинеска.

\section{2. ПРЕОБРАЗОВАНИЯ ДАРБУ}

2.1. Теорема о приводимости. Рассматривая преобразования Дарбу спектральной задачи $L \psi=\lambda \psi$ для дифференциального оператора $L$ порядка $m$ удобно полагать, что

$$
L=D^{m}+u^{(1)} D^{m-2}+\cdots+u^{(m-2)} D+u^{(m-1)} .
$$

Для дифференциальных операторов обшего вида әлементарные преобразования Дарбy, переводящие решения $L \psi=\lambda \psi$ в решения $\hat{L} \hat{\psi}=\lambda \hat{\psi}$, задаются формулой $\hat{\psi}=$ $a(x) \psi_{x}+b(x) \psi$. Замена независимой переменной и простое калибровочное преобразование $L \mapsto g^{-1} L g$, приводящие $L$ к виду (2), приводят и элементарные преобразования Дарбу к каноническому виду:

$$
\hat{\psi}=\psi_{x}-f \psi, \quad f=D \ln \varphi, \quad L \varphi=\mu \varphi .
$$

Предполагается, естественно, что преобразованный оператор $\hat{L}$ имеет тот же вид (2). Очевидно, что элементарное преобразование Дарбу зависит от выбора частного решения $\varphi$ исходной спектральной задачи. Ниже мы будем записывать формулу (3) в операторном виде:

$$
\hat{L}(D-f)=(D-f) L .
$$

ОПРеДЕЛЕНИЕ 1. Дифференциальный оператор $M$ называется оператором преобразования из $L$ в $\hat{L}$, если

$$
M L=\hat{L} M .
$$

Приведем известное доказательство приводимости преобразований Дарбу, основанное на этом определении. 
Теорема. Любой оператор преобразования $M$ из $L$ в $\hat{L}$ разлагается в произведение операторов преобразования первого порядка.

ДокАЗАТЕльСтво. Для доказательства достаточно установить разрешимость следуюшей задачи "об обшей собственной функции":

$$
M \psi=0, \quad(L-\lambda) \psi=0 .
$$

Действительно, при заданном решении $\psi=\varphi, \lambda=\mu$ задачи (5) и $f=D \ln \varphi$ мы имеем

$$
M=\widetilde{M}(D-f), \quad L-\mu=\tilde{L}(D-f) .
$$

Подставив эти разложения в уравнение (4), находим

$$
\hat{L} M=\hat{L} \widetilde{M}(D-f)=\widetilde{M} \tilde{L}(D-f) \Rightarrow \hat{L} \widetilde{M}=\widetilde{M} \tilde{L}
$$

Мы можем теперь заменить пару дифференциальных операторов $M, L$, удовлетворяюших уравнению (4), на пару $\widetilde{M}, \tilde{L}$. При этом $\widetilde{M}$, являясь оператором преобразования, имеет порядок меньший, чем исходньй, и можно воспользоваться индукцией относительно порядка оператора преобразования $M$.

Наконец, так как $M \psi=0 \Rightarrow M L \psi=\hat{L} M \psi=0$, то разрешимость задачи (5) становится очевидной, если рассмотреть сужение оператора $L$ на конечномерное инвариантное подпространство $V=\operatorname{Ker} M$. Таким образом, в качестве $\mu$ можно выбрать любое собственное значение конечномерного линейного оператора $\left.L\right|_{V}$.

В силу теоремы оператор преобразования $M$ порядка $n$ представим в виде суперпозиции операторов преобразования первого порядка $D-f_{j}$ :

$$
M=\left(D-f_{n}\right)\left(D-f_{n-1}\right) \ldots\left(D-f_{1}\right) .
$$

Это приводит к последовательности операторов (2) $L_{1}=L, L_{2}, \ldots, L_{n+1}=\hat{L}$, связанных соотношениями

$$
L_{j+1}\left(D-f_{j}\right)=\left(D-f_{j}\right) L_{j}, \quad f_{j}=D \ln \varphi_{j}, \quad L_{j} \varphi_{j}=\mu_{j} \varphi_{j}, \quad j=1, \ldots, n .
$$

При $m=2$ мы находим, что $L_{j}=\left(D+f_{j}\right)\left(D-f_{j}\right)+\mu_{j}$ и что соотношения (7) эквивалентны следуюшей системе уравнений для $f_{1}, \ldots, f_{n}$ :

$$
f_{j, x}+f_{j+1, x}=f_{j}^{2}-f_{j+1}^{2}+\alpha_{j}
$$

Здесь $j=1, \ldots, n-1$ и некоторые из параметров $\alpha_{j}=\mu_{j}-\mu_{j+1}$ могут равняться нулю. Отметим, что из доказательства теоремы вытекает, что случай $\alpha_{1}=\cdots=\alpha_{n}=0$ соответствует нильпотентности оператора $\left.(L-\mu)\right|_{V}$. Тем не менее и в этом случае жордановой клетки каждый из операторов $D-f_{j}$ имеет канонический вид (3) и не требует привлечения присоединенных собственных функций. 
В случае $m=3$ и $L=D^{3}+u D+v$ операторные соотношения (7) дают

$$
\begin{aligned}
L_{j}=D^{3}+u_{j} D+v_{j} \Rightarrow & u_{j+1}=u_{j}+3 f_{j, x}, \quad v_{j+1}=v_{j}+3 f_{j, x x}+3 f_{j} f_{j, x}+u_{j, x}, \\
& f_{j, x x}+3 f_{j} f_{j, x}+f_{j}^{3}+u_{j} f_{j}+v_{j}=\mu_{j} .
\end{aligned}
$$

Эти уравнения удается записать в более компактном виде:

$$
\begin{aligned}
& \left(q_{j-1}+q_{j}+q_{j+1}\right)_{x x}= \\
& \quad=3\left(q_{j}-q_{j-1}\right) q_{j-1, x}-3\left(q_{j+1}-q_{j}\right) q_{j+1, x}+\left(q_{j}-q_{j-1}\right)^{3}+\left(q_{j}-q_{j+1}\right)^{3}+\alpha_{j},
\end{aligned}
$$

где

$$
\alpha_{j}=\mu_{j}-\mu_{j-1}, \quad u_{j}=3 q_{j, x}, \quad f_{j}=q_{j+1}-q_{j} .
$$

В обшем случае $m \geqslant 2$ удобно, используя разложение (6), ввести дискретный аналог рассматриваемой спектральной задачи. Для этого достаточно (ср. [1]) заменить $D^{k} \psi$ в уравнении $L \psi=\lambda \psi$ степенями оператора сдвига $T$ :

$$
T^{k} \psi=\left(D-f_{k}\right) \ldots\left(D-f_{1}\right) \psi, \quad k=1,2, \ldots, m .
$$

Полученное таким нехитрым способом уравнение имеет следующий вид:

$$
L^{\mu} \psi=\lambda \psi, \quad L^{\mu}=T^{m}+a^{(1)} T^{m-1}+a^{(2)} T^{m-2}+\cdots+a^{(m-1)} T+\mu,
$$

причем коэффишиенты $a^{(k)}$ являются дифференциальными полиномами от $f_{1}, \ldots, f_{m}$ и, в частности,

$$
a^{(1)}=f_{1}+f_{2}+\cdots+f_{m} .
$$

Например, при $m=2$ мы получаем $L^{\mu}=T^{2}+g T+\mu, g=f_{1}+f_{2}$.

Окончательную форму полученной "разностной” спектральной задачи мы постулируем в виде

$$
L_{j}^{\mu} \psi_{j}=\lambda \psi_{j} \Longleftrightarrow \psi_{j+m}+a_{j}^{(1)} \psi_{j+m-1}+\cdots+a_{j}^{(m-1)} \psi_{j+1}+\mu_{j} \psi_{j}=\lambda \psi_{j}
$$

и рассматриваем (11) как дискретный аналог исходной спектральной задачи $L \psi=\lambda \psi$.

ОПРЕДЕЛЕНИЕ 2. Одеваюшей цепочкой для спектральной задачи $L \psi=\lambda \psi$ называются условия совместности уравнения (11) с уравнением $\psi_{j, x}=\psi_{j+1}+f_{j} \psi_{j}$.

Стандартный анализ показывает, что условия совместности элементарного преобразования Дарбу (3) и дискретного аналога $L_{j}^{\mu} \psi_{j}=\lambda \psi_{j}$ рассматриваемой спектральной задачи сводятся к следуюшим уравнениям:

В этих уравнениях

$$
\begin{aligned}
& a_{j, x}^{(1)}=a_{j}^{(1)}\left(f_{j}-f_{j+m-1}\right)+a_{j+1}^{(2)}-a_{j}^{(2)}, \\
& a_{j, x}^{(2)}=a_{j}^{(2)}\left(f_{j}-f_{j+m-2}\right)+a_{j+1}^{(3)}-a_{j}^{(3)}, \\
& a_{j, x}^{(m-1)}=a_{j}^{(m-1)}\left(f_{j}-f_{j+1}\right)+\alpha_{j} .
\end{aligned}
$$

$$
\alpha_{j}=\mu_{j+1}-\mu_{j}, \quad a_{j}^{(1)}=f_{j}+f_{j+1}+\cdots+f_{j+m} .
$$

Возврашаясь к операторам преобразования, мы получаем следующее обобщение результата работы [2]: 
ПРЕДЛОЖЕНИЕ 1. Рассмотрим периодическое $\mathbb{Z}_{n}$-решение одевающей цепочки дифференциальных уравнений (12). Пусть

$$
\gamma=\alpha_{1}+\cdots+\alpha_{n}, \quad M=\left(D-f_{n}\right) \ldots\left(D-f_{1}\right)
$$

u $L=L_{1}, \quad \hat{L}=L_{n+1}$ в соответствующей чепочке операторов (7). Тогда при любом $n>0$ оператор $M$ является оператором преобразования из $L$ в $\hat{L} u \hat{L}=$ $L+\gamma$.

Опуская доказательство, которое аналогично приведенному в работе [2] для одевающей цепочки (8), отметим, что равенство $\hat{L}=L+\gamma$ следует из соотношений

$\mu_{2}-\mu_{1}=\alpha_{1}, \ldots, \quad \mu_{n+1}-\mu_{n}=\alpha_{n} \Rightarrow \mu_{1}-\mu_{n+1}=\alpha_{1}+\cdots+\alpha_{n}=\gamma \Rightarrow \hat{L}=L+\gamma$

Заметим еше, что при доказательстве предполагается однозначная разрешимость линейной системы алгебраических уравнений относительно $f_{i}$ :

$$
f_{i}+f_{i+1}+\cdots+f_{i+m}=a_{i}^{(1)}, \quad i \in \mathbb{Z}_{n}
$$

Это условие налагает определенные ограничения на целые числа $m$ и $n$.

Совпадение одеваюшей цепочки (12) при $m=2$ с цепочкой (8) очевидно. В случае $m=3$ одеваюшая цепочка (12) сводится к следуюшим уравнениям для $a_{j}=a_{j}^{(1)}$, $b_{j}=a_{j}^{(2)}$

$$
\begin{aligned}
a_{j} & =f_{j}+f_{j+1}+f_{j+2}, \\
a_{j, x} & =a_{j}\left(f_{j}-f_{j+2}\right)+b_{j+1}-b_{j}, \\
b_{j, x} & =b_{j}\left(f_{j}-f_{j+1}\right)+\alpha_{j} .
\end{aligned}
$$

Переписав второе из этих уравнений в виде

$$
\left(1+T+T^{2}\right)\left(f_{j, x}\right)=(1-T)\left(f_{j}^{2}+f_{j} f_{j+1}+f_{j+1}^{2}-b_{j}\right)
$$

мы приходим к уравнениям (10) в результате замены

$$
f_{j}=q_{j+1}-q_{j}, \quad b_{j}=\left(1+T+T^{2}\right) q_{j, x}+f_{j}^{2}+f_{j} f_{j+1}+f_{j+1}^{2} .
$$

Заметим, что аналогичное преобразование, т.е. замена переменных $f_{j}=q_{j+1}-q_{j}$ в уравнениях одеваюшей цепочки (8) приводит эти уравнения к виду

$$
\left(q_{j}+q_{j+1}\right)_{x}=\beta_{j}-\left(q_{j}-q_{j+1}\right)^{2}, \quad \alpha_{j}=\beta_{j+1}-\beta_{j}
$$


2.2. Уравнение $A B-B A=\alpha A+\beta B$. Непосредственным следствием сформулированного выше предложения 1 является разрешимость задачи о паре дифференциальных операторов $A$ и $B$. Заметим, что ограничения на порядки этих операторов, связанные с условием разрешимости линейной системы (13), можно было бы ослабить. Впрочем, мы рассмотрим здесь только простейший случай, при котором эти условия выполняются автоматически.

Рассматривая пару операторов $A$ и $B$ порядка 2 и 3, удовлетворяющих уравнению

$$
A B-B A=\alpha A+\beta B
$$

нужно иметь в виду, что при $\beta \neq 0$ замена $B$ на $\widetilde{B}=\alpha A+\beta B$ приводит нас к случаю $\alpha=0$. При этом в силу предложения 1 применима цепочка (8) (см. ниже). В случае $\beta=0 \mathbb{Z}_{2}$-решения цепочки (10) определяются уравнениями

$$
\begin{aligned}
& \left(2 q_{1}+q_{2}\right)_{x x}=6 q_{1, x}\left(q_{2}-q_{1}\right)+2\left(q_{2}-q_{1}\right)^{3}+\alpha_{1}, \\
& \left(2 q_{2}+q_{1}\right)_{x x}=6 q_{2, x}\left(q_{1}-q_{2}\right)+2\left(q_{1}-q_{2}\right)^{3}+\alpha_{2} .
\end{aligned}
$$

Эти уравнения и дают в силу предложения 1 пару операторов, удовлетворяющих уравнению (15):

$$
\begin{gathered}
A=(D+f)(D-f), \quad B=D^{3}+u D+v, \quad \alpha=\alpha_{1}+\alpha_{2}, \\
f=q_{2}-q_{1}, \quad u=3 q_{1, x}, \quad v=\frac{1}{2} u_{x} .
\end{gathered}
$$

Указанные уравнения для $q_{1}, q_{2}$ допускают первый интеграл

$$
\left(q_{1}+q_{2}\right)_{x}+\left(q_{1}-q_{2}\right)^{2}=a(x), \quad 3 a^{\prime}=\alpha,
$$

и сводятся благодаря этому интегралу к уравнению второго порядка для $f=q_{2}-q_{1}$ :

$$
f_{x x}=2 f^{3}-6 a(x) f+\alpha_{2}-\alpha_{1} .
$$

Уравнение (16) является, очевидно, вторым из уравнений Пенлеве.

Периодическое $\mathbb{Z}_{3}$-замыкание одевающей цепочки для уравнения Шредингера приводит нас к следующей системе уравнений первого порядка для $g_{j}=f_{j}+f_{j+1}, j \in \mathbb{Z}_{3}$ :

$$
g_{1, x}=g_{1}\left(g_{3}-g_{2}\right)+\alpha_{1}, \quad g_{2, x}=g_{2}\left(g_{1}-g_{3}\right)+\alpha_{2}, \quad g_{3, x}=g_{3}\left(g_{2}-g_{1}\right)+\alpha_{3} .
$$

В этом случае мы имеем

$$
A=\left(f_{1}+D\right)\left(f_{1}-D\right), \quad B=\left(D-f_{3}\right)\left(D-f_{2}\right)\left(D-f_{1}\right) \quad \Rightarrow \quad A B=B(A+\gamma),
$$

где $\gamma=\alpha_{1}+\alpha_{2}+\alpha_{3}$. Как и в предыдущем случае, первый интеграл

$$
g_{1}+g_{2}+g_{3}=a(x), \quad a^{\prime}=\gamma,
$$


позволяет понизить порядок системы и свести ее к четвертому уравнению Пенлеве. Точнее, как нетрудно проверить, каждая из трех функций $g_{j} \equiv g, j=1,2,3$, удовлетворяет уравнению второго порядка

$$
2 g g_{x x}-g_{x}^{2}=g^{2}\left(3 g^{2}-4 \sigma g+\sigma^{2}+2 \alpha_{j+2}+2 \alpha_{j+1}\right)-\alpha_{j}^{2} .
$$

В частности, при $g_{3}=0$ уравнения (17) сводятся к уравнению Риккати

$$
g_{x}+g^{2}=\gamma x g+\alpha,
$$

где $g \equiv g_{2}, \alpha \equiv \alpha_{2}$ и $g_{1}=\gamma x-g$. Подстановка $g=y^{\prime} / y$ переводит это уравнение Риккати в линейное уравнение, определяюшее функции Эрмита. Дальнейшее упрощение происходит при $g_{2}=g_{3}=0$, когда система (17) сводится к уравнению $g_{1, x}=\gamma$. Порядок оператора $B$ в (18) можно понизить, заметив, что в обшем случае (15)

$$
B=A B_{1} \quad \Leftrightarrow \quad A B_{1}-B_{1} A=\alpha+\beta B_{1} .
$$

В рассматриваемом примере $f_{1}=f_{2}=-f_{3}=g_{1} / 2$, и поэтому $B=\left(D-f_{3}\right)\left(D-f_{2}\right) \times$ $\left(D-f_{1}\right)=A\left(D-f_{1}\right)$. Таким образом, в случае $g_{2}=g_{3}=0$ уравнение (18) можно переписать в виде

$$
L(f-D)=(f-D) L+\gamma(f-D), \quad L=u-D^{2} \Leftrightarrow 2 f_{x}=\gamma, \quad u_{x}=\gamma f .
$$

Эта формула и полученная из нее формальным сопряжением позволяют, как известно, вычислить спектр оператора $L=x^{2}-D^{2}$, соответствующего квантовому гармоническому осциллятору. В силу предложения 1 случай $(20)$ соответствует $\mathbb{Z}_{1}$-периодическим решениям одеваюшей цепочки для оператора Шредингера.

2.3. Обобщения цепочки Вейсса. Впервые одевающая цепочка (12), а точнее ее частный случай, появилась в работе Вейсса [3] в следуюшем виде:

$$
\frac{a_{j, x}}{a_{j}}+\frac{a_{j+1, x}}{a_{j+1}}+\cdots+\frac{a_{j+m-1, x}}{a_{j+m-1}}=a_{j}-a_{j+m-1} .
$$

Эта отвечаюшая жордановой клетке (см. замечание после теоремы) цепочка получается из системы (12) в результате редукции

$$
a_{j}^{(m-1)}=\cdots=a_{j}^{(2)}=0, \quad a_{j}^{(1)}=a_{j}=f_{j}+f_{j+1}+\cdots+f_{j+m} .
$$

Очевидно, одеваюшая цепочка при $m>3$ допускает и другие редукции, понижаюшие порядок системы (12):

$$
a_{j}^{(m-1)}=\cdots=a_{j}^{(m-k)}=0, \quad 1<k \leqslant m-3 .
$$


Заметим, что все эти редукции легко формулируются в терминах вспомогательной линейной задачи (см. определение 2). В частности, цепочке (21) соответствует спектральная задача

$$
\psi_{j+m}+a_{j} \psi_{j+m-1}=\lambda \psi_{j}, \quad \psi_{j, x}=\psi_{j+1}+f_{j} \psi_{j},
$$

которая получается из спектральной задачи (11) в результате редукции $(22)$ при $\mu_{j}=0$.

В недавней работе [4] рассматривалась следуюшая модификация спектральной задачи $(23)$ :

$\psi_{j+3}+a_{j} \psi_{j+1}+b_{j} \psi_{j-1}=\lambda \psi_{j}, \quad \psi_{j, x}=\psi_{j+2}+\left(f_{j}+f_{j+1}\right) \psi_{j}, \quad a_{j}=f_{j}+f_{j+1}+f_{j+2}$.

Соответствуюшие уравнения цепочки

$$
\begin{aligned}
\left(f_{j-1}+f_{j}+f_{j+1}\right)_{x} & =\left(f_{j-1}+f_{j}+f_{j+1}\right)\left(f_{j-1}-f_{j+1}\right)+b_{j+1}-b_{j-1}, \\
b_{j, x} & =b_{j}\left(f_{j+1}-f_{j-1}\right)
\end{aligned}
$$

сводятся, очевидно, к уравнениям (21) при $m=3$ редукцией $b=0$.

Наконец, пара Лакса $L \psi=\lambda \psi, \psi_{x}=A \psi($ ср. [5]), где

$$
L=T^{m}-\left(f+\cdots+f_{m-1}\right) T^{-k}, \quad A=T^{k+m}-\left(f+\cdots+f_{k+m-1}\right), \quad k+m>0,
$$

приводит к еше одной интересной модификации цепочки Вейсса (21):

$\left(f_{j}+\cdots+f_{j+m-1}\right)_{x}=\left(f_{j}+\cdots+f_{j+m-1}\right)\left(f_{j-k}+\cdots+f_{j-1}-f_{j+m}-\ldots-f_{j+k+m-1}\right)$.

Случай $k=1-m$ дает в точности редукцию Вейсса, рассмотренную выше, случай $m=$ 1 приводит к цепочке Богоявленского [6].

\section{3. ГАМИЛЬТОНОВА ТЕОРИЯ ПБ}

3.1. Канонические преобразования. Мы рассмотрим здесь альтернативный подход к одеваюшим цепочкам, в котором место преобразований Дарбу занимают ПБ для нелинейных интегрируемых уравнений. Большая часть примеров связана с уравнениями из известного списка [7] систем вида

$$
\mathbf{u}_{t}=A(\mathbf{u}) \mathbf{u}_{x x}+B\left(\mathbf{u}, \mathbf{u}_{x}\right), \quad \operatorname{det} A \neq 0, \quad \mathbf{u}=\left(u^{1}, u^{2}\right),
$$

обладаюших высшими законами сохранения. В частности, этот класс содержит НУШ и уравнение Буссинеска

$$
q_{t t}+q_{x x x x}+q_{x} q_{x x}=0 .
$$

Отметим, что первоначально одеваюшая цепочка в виде (10) возникла именно при рассмотрении ПБ для этого уравнения. Остальные примеры связаны с уравнениями типа КдФ и Савады-Котера. 
Как цепочки, так и непрерывные уравнения обладают лагранжевой структурой. Грубо говоря, нас интересует вопрос, как связаны эти две структуры. Грубо же, можно ответить так: во всех случаях сдвиг в цепочке является каноническим преобразованием, сохраняющим гамильтонову структуру уравнения. С некоторыми оговорками можно сказать и наоборот: уравнение в частных производных задает вариационную симметрию цепочки.

Сначала обсудим общую схему на примере гамильтоновых систем (24). Эта теория является достаточно обшей и охватывает широкий класс вариационных задач вида

$$
\delta \int d t d x \Phi\left(q_{t}, q_{x x}, q_{x}, q\right)=0
$$

В частности, лагранжианы с плотностями

$\Phi=\left(q_{t}+q_{x x}\right)^{2}+q_{x}^{3}, \quad \Phi=\frac{1}{2}\left(q_{t}+q_{x x}-q_{x}^{2}\right)^{2}, \quad \Phi=\frac{\left(q_{t}+q_{x x}-\frac{1}{2} R^{\prime}(q)\right)^{2}}{4\left(q_{x}^{2}-R(q)\right)}+\frac{1}{12} R^{\prime \prime}(q)$

отвечают соответственно уравнению Буссинеска, НУШ и анизотропной модели Ландау-Лифшица. Плотность $H[p, q]=H\left(p, q_{x x}, q_{x}, q\right)$ гамильтониана связана с плотностью лагранжиана $\Phi$ стандартными формулами

$$
H=q_{t} \Phi_{q_{t}}-\Phi, \quad \Phi=p H_{p}-H \quad\left(p=\Phi_{q_{t}}\right)
$$

Отметим, что подстановка

$$
p \rightarrow p+\alpha q_{x x}
$$

хотя и меняет вид гамильтониана, не отражается на исходном $q$-уравнении.

В излагаемой теории роль цепочки преобразований Дарбу играет цепочка ПБ

$$
\ldots\left(p_{n-1}, q_{n-1}\right) \rightarrow\left(p_{n}, q_{n}\right) \rightarrow\left(p_{n+1}, q_{n+1}\right) \ldots,
$$

переводящих решение гамильтоновой динамической системы

$$
q_{t}=\frac{\delta H[p, q]}{\delta p}, \quad p_{t}=-\frac{\delta H[p, q]}{\delta q}
$$

снова в решение.

Аналог элементарных преобразований Дарбу (см. предыдуший раздел) определяется как преобразование $(p, q) \rightarrow(\hat{p}, \hat{q})$ с помощью производящей функции $F[q, \hat{q}]$ :

$$
p=\frac{\delta F[q, \hat{q}]}{\delta q}, \quad \hat{p}=-\frac{\delta F[q, \hat{q}]}{\delta \hat{q}}
$$


ОПРЕДЕЛЕниЕ 3 . Функция $F[q, \hat{q}]=F\left(q, q_{x}, \hat{q}, \hat{q}_{x}\right)$ называется производящей функцией канонического ПБ для динамической системы (29), если сушествует функция $\sigma[q, \hat{q}]$ такая, что

$$
\widehat{H}[\hat{p}, \hat{q}]-H[p, q]=\frac{d}{d x} \sigma[q, \hat{q}],
$$

где $p, \hat{p}$ определены формулами (30).

В силу этого определения и соотношений

$$
p_{n}=\frac{\delta F\left[q_{n}, q_{n+1}\right]}{\delta q_{n}}=-\frac{\delta F\left[q_{n-1}, q_{n}\right]}{\delta q_{n}} \Rightarrow \frac{\delta F\left[q_{n}, q_{n+1}\right]}{\delta q_{n}}+\frac{\delta F\left[q_{n-1}, q_{n}\right]}{\delta q_{n}}=0
$$

производяшая функция приводит нас к лагранжевой форме цепочки и задает плотность $F\left[q_{n}, q_{n+1}\right]$ лагранжиана:

$$
\delta \int d x \sum_{n} F\left[q_{n}, q_{n+1}\right]=0 .
$$

ПримеР 1. Для гамильтониана $H=p^{2}+p\left(q_{x}^{2}-q_{x}\right)$, соответствующего второму из примеров (26), производяшая функция

$$
F=-q_{x}^{2}+f(\hat{q}-q), \quad f^{\prime \prime}+f^{\prime}=0,
$$

приводит нас к лагранжиану для цепочки Тоды.

Подставляя выражения (30) для импульсов в (31), мы получим определяющее уравнение для производящей функции $F$ и гамильтониана $H$, к разрешению которого сводится задача классификации. Разумеется, в полном объеме эта задача является весьма сложной, и здесь мы ограничимся лишш некоторыми подклассами уравнений. Например, в качестве дополнительного условия, сильно облегчающего задачу, можно считать, что гамильтониан не зависит явно от $q$ (что отвечает инвариантности уравнений относительно сдвигов $q \rightarrow q+$ const). Другим дополнительным условием может служить предположение о квадратичности гамильтониана по $p$ :

$$
H=G\left(q_{x}, q\right) p^{2}+\left(A\left(q_{x}, q\right)-q_{x x}\right) p+B\left(q_{x}, q\right) .
$$

Соответствуюшие динамические системы являются лагранжевыми,

$$
L=\int d x\left(\frac{1}{2 G}\left(A-q_{x x}-q_{t}\right)^{2}-2 B\right),
$$

и описывают ньютоновскую динамику одномерного объекта (струны) с плотностью $\rho=G^{-1}$ и дисперсией $\omega= \pm k^{2}$. В данном случае определяющее уравнение сводится в главном к системе

$$
F_{a a}\left(G F_{a a}+1\right)=\widehat{G} F_{a b}^{2}, \quad G F_{a b}^{2}=F_{b b}\left(\widehat{G} F_{b b}-1\right), \quad\left(G F_{a a}-\widehat{G} F_{b b}+1\right) F_{a b}=0,
$$

где использованы обозначения $q_{x}=a, \hat{q}_{x}=b$. 
3.2. Уравнения типа НУШ и цепочки типа Тоды. Уравнения (34) приводят к сушественно различным типам систем (33) при $F_{a b}=0$ и $F_{a b} \neq 0$. В случае $F_{a b}=0$ получаем обобщенные цепочки Тоды (см. [8]-[11]), при этом производящая функция имеет вид

$F=f(a, q)+a \alpha(q, \hat{q})+b \beta(q, \hat{q})+\gamma(q, \hat{q}), \quad \sigma=r(a, q)+a b h(q, \hat{q})+\gamma_{q} a-\gamma_{\hat{q}} b+\rho(q, \hat{q})$

причем $G f_{a a}=-1, r_{a}=f_{q}-a f_{a q}-A f_{a a}, h=\beta_{q}-\alpha_{\hat{q}}$.

Подставляя (35) в (31), имеем

$$
\begin{aligned}
a^{2} b h_{q} & +a b^{2} h_{\hat{q}}+a\left(r_{q}+\gamma_{q q} a+\rho_{q}\right)+b\left(\rho_{\hat{q}}-\gamma_{\hat{q} \hat{q}} b\right)= \\
= & \left(f_{a q} a-f_{q}-\gamma_{q}-b h\right)\left[G\left(f_{a q} a-f_{q}-\gamma_{q}-b h\right)-A\right]+ \\
& +B-\widehat{B}-\left(a h-\gamma_{\hat{q}}\right)\left[\hat{G}\left(a h-\gamma_{\hat{q}}\right)+\hat{A}\right]
\end{aligned}
$$

откуда легко следует, что $G_{a a a}=\widehat{G}_{b b b}=0$.

ПРимеР 2. В качестве несложного упражнения разберем подробно случай без явной зависимости от $q$. В этом случае

$$
H=p q_{x x}+\varepsilon_{0} p^{2} q_{x}^{2}+\varepsilon_{1} p q_{x}^{2}+\varepsilon_{2} p^{2} q_{x}+\varepsilon_{3} p^{2}+\varepsilon_{4} p q_{x}+\varepsilon_{5} q_{x}^{2}+\varepsilon_{6} p,
$$

производящая функция не зависит от $\hat{z}$ и уравнение (31) дает

$$
F(z, y)=W(z)+z V(y)+U(y), \quad y=\hat{q}-q, \quad z \equiv q_{x}
$$

Импульсы задаются формулами

$$
p=-c q_{x x}-a \hat{q}_{x}-b, \quad \hat{p}=-a q_{x}-b,
$$

где $a=V^{\prime}, b=U^{\prime}, c=W^{\prime \prime}$. Условие $\widehat{H}-H \in \operatorname{Im} D_{x}$ эквивалентно следующим уравнениям на эти функции:

$$
\frac{1}{c}=\varepsilon_{0} q_{x}^{2}+\varepsilon_{2} q_{x}+\varepsilon_{3}, \quad a^{\prime}=\varepsilon_{2} a^{2}-2 \varepsilon_{0} a b+\varepsilon_{1} a, \quad b^{\prime}=\varepsilon_{3} a^{2}-\varepsilon_{0} b^{2}+\varepsilon_{1} b-\varepsilon_{5} .
$$

Система на $a$ и $b$ допускает первый интеграл,

$$
-\varepsilon_{5}+\varepsilon_{1} b-\varepsilon_{0} b^{2}+\varepsilon_{2} a b-\varepsilon_{3} a^{2}=a \mu,
$$

и решается в элементарных функциях. В результате получается известный список цепочек типа Тоды (случай $a=0)$ и релятивистской цепочки Тоды $(a \neq 0)$. Постоянная интегрирования $\mu$ не входит в уравнения в частных производных (которые определяются только параметрами $\varepsilon$ ), и при итерации ПБ ее можно выбирать независимо на каждом шаге, как и спектральный параметр в преобразовании Дарбу. 
ПрЕДЛОЖЕнИЕ 2. В общем случае $F_{a b}=0$

$$
F\left(q_{n, x}, q_{n+1}, q_{n}\right)=W\left(q_{n, x}, q_{n}\right)+q_{n, x} V\left(q_{n+1}, q_{n}\right)+U\left(q_{n+1}, q_{n}\right) .
$$

В частности, для модели Ландау-Лифшшица (см. (26)) кинетическая энергия $W=$ $W(z, q)$ определяется слагаемым

$$
W=\frac{1}{2 \sqrt{R}}[(\sqrt{R}+z) \ln (\sqrt{R}+z)+(\sqrt{R}-z) \ln (\sqrt{R}-z)], \quad W_{z z}=\frac{1}{R(q)-z^{2}},
$$

а функции $U$ и $V$ задаются формулами

$$
\begin{aligned}
V & =0, & U & =-\ln \left(q_{1}-q\right), \\
V_{q_{1}} & =-\frac{1}{2 r\left(q_{1}, q\right)}, & U & =\frac{1}{2} \ln r\left(q_{1}, q\right),
\end{aligned}
$$

где в обоих случаях $R(u)$ есть многочлен не выше четвертой степени, $r(u, v)$ - симметрический полином степени не вьше 2 по каждой переменной, причем $R$ является его дискриминантом:

$$
R(u)=r_{v}^{2}-2 r r_{v v}, \quad r(u, v)=r(v, u), \quad r_{u u u}=0 .
$$

Гамильтонова форма соответствуюших цепочек (см. [10])

$$
u_{n, x}=\frac{2 h\left(u_{n}, v_{n}\right)}{u_{n+1}-v_{n}}+h_{n, v_{n}}, \quad v_{n, x}=\frac{2 h\left(u_{n}, v_{n}\right)}{u_{n}-v_{n-1}}-h_{n, u_{n}}
$$

непосредственно связана [12] с известной $X Y Z$-цепочкой Склянина [13].

3.3. Уравнения типа Буссинеска. В случае $F_{a b} \neq 0$, уравнения (34) эквивалентны переопределенной системе

$$
F_{a a} F_{b b}=F_{a b}^{2}, \quad G(a) F_{a a}-\widehat{G}(b) F_{b b}+1=0 .
$$

Обозначим $y=F_{a a}$, тогда $F_{b b}=(G y+1) / \widehat{G}, F_{a b}^{2}=y(G y+1) / \widehat{G}$. Из условий совместности $F_{b b a}=F_{a b b}, F_{a b a}=F_{a a b}$ нетрудно получить выражения для $y_{a}, y_{b}$ (определитель линейной системы не равен нулю). Условие совместности $y_{a b}=y_{b a}$ оказывается эквивалентным равенству $\widehat{G}_{b b}=G_{a a}$, т.е. функции $\widehat{G}$ и $G$ квадратичны, как и в предыдушем случае. Далее, уравнения на функцию $y$ удается сильно упростить в результате подстановки

$$
u=\widehat{G} G+\frac{\widehat{G}}{4 y(G y+1)}=\widehat{G} G+\frac{1}{4 F_{a b}^{2}}, \quad u_{a}^{2}=\widehat{G}_{b}^{2} u, \quad u_{b}^{2}=G_{a}^{2} u .
$$

Если $u \neq 0$, то замена $v=\sqrt{u}$ дает $2 v_{a}=\widehat{G}_{b}, 2 v_{b}=G_{a}$, и мы получаем решение в виде

$$
\begin{gathered}
G=g_{2} a^{2}+2 g_{1} a+g_{0}, \quad \widehat{G}=g_{2} b^{2}+2 \hat{g}_{1} b+\hat{g}_{0}, \\
v=g_{2} a b+\hat{g}_{1} a+g_{1} b+c, \quad w=v^{2}-\widehat{G} G, \\
F_{a a}=-\frac{1}{2 G}\left(\frac{v}{\sqrt{w}}+1\right), \quad F_{b b}=-\frac{1}{2 \widehat{G}}\left(\frac{v}{\sqrt{w}}-1\right), \quad F_{a b}=\frac{1}{2 \sqrt{w}},
\end{gathered}
$$

откуда $F$ находится интегрированием. Постоянную интегрирования $c$ следует выбирать так, чтобы $w \neq 0$.

В случае $u=0$ имеем $2 F_{a a}=1 / G, 2 F_{b b}=1 / \widehat{G}, 4 F_{a b}^{2}=-1 / \widehat{G} G$, откуда следует, что функции $G$ и $\widehat{G}$ постоянны, а $F$ квадратична. 
ПримеР 3. В простейшем случае $G=\widehat{G}=1$ мы приходим к производяшей функции

$$
F[q, \hat{q}]=(1+c) q_{x}^{2}+4 q_{x} \hat{q}_{x}+(1-c) \hat{q}_{x}^{2}+3\left(q_{x}+\hat{q}_{x}\right)(q-\hat{q})^{2}+(q-\hat{q})^{4}+4 \mu(q-\hat{q})
$$

и гамильтониану

$$
H=p^{2}+4 c q_{x x} p+4\left(c^{2}+3\right) q_{x x}^{2}-48 q_{x}^{3},
$$

где произвол в выборе постоянной $c$ соответствует преобразованию (27):

$$
\begin{aligned}
& p=-2(1+c) q_{x x}-4 \hat{q}_{x x}+12(q-\hat{q}) \hat{q}_{x}+4(q-\hat{q})^{3}+4 \mu, \\
& \hat{p}=+2(1-c) \hat{q}_{x x}+4 q_{x x}+12(q-\hat{q}) q_{x}+4(q-\hat{q})^{3}+4 \mu .
\end{aligned}
$$

Легко видеть, что соответствуюшая цепочка совпадает с цепочкой (10), а соответствуюшая система (29) эквивалентна, с точностью до растяжений, уравнению Буссинеска (25).

ПримеР 4. Вводя новые переменные $Q=\alpha q+\beta \hat{q}, \hat{Q}=\beta q+\alpha \hat{q}$, можно получить систему Михайлова-Шабата

$$
Q_{t}=-\gamma Q_{x x}+\mu \widehat{Q}_{x}(Q-\widehat{Q})+\nu(Q-\widehat{Q})^{3}, \quad \widehat{Q}_{t}=\gamma \widehat{Q}_{x x}+\mu Q_{x}(Q-\widehat{Q})+\nu(Q-\widehat{Q})^{3},
$$

причем

$$
\beta=\alpha \rho, \quad \rho^{2}-\rho+1=0, \quad \gamma=3\left(\rho-\frac{1}{\rho}\right), \quad \mu=-\frac{2}{3} \frac{1}{\alpha-\beta}, \quad \nu=-\frac{2}{27} \frac{\alpha+\beta}{(\alpha-\beta)^{3}} .
$$

ПримеР 5. В заключение приведем еше один пример канонического преобразования, показываюший, что предположение квадратичности по $p$ не является обязательным $\left(\omega^{3}=1\right)$ :

$$
\begin{gathered}
H=p_{x} q_{x}+p^{3}+q_{x}^{3} \rightarrow \widehat{H}=-\hat{p}_{x} \hat{q}_{x}+\hat{p}^{3}+\hat{q}_{x}^{3}, \\
F=\omega \hat{q}_{x} q+f(q, \hat{q}), \quad p=\omega \hat{q}_{x}+f_{q}, \quad \hat{p}=\omega q_{x}-f_{\hat{q}} .
\end{gathered}
$$

Используя выражения (46) для гамильтонианов и импульсов и требование $\delta(\widehat{H}-H)=0$, получаем уравнения на $f$ :

$$
f_{\hat{q} \hat{q}}-3 \omega^{2} f_{q}=0, \quad f_{q q}+3 \omega^{2} f_{\hat{q}}=0, \quad\left(f_{\hat{q}}^{2}\right)_{\hat{q}}+\left(f_{q}^{2}\right)_{q}=0, \quad f_{q}^{3}+f_{\hat{q}}^{3}=\text { const } .
$$

Решая систему (47), получаем лагранжиан цепочки ПБ системы (46):

$$
L=\sum_{n}\left[\omega_{n} q_{n+1, x} q_{n}+C_{n} e^{\frac{3}{\omega_{n}}\left(\Omega_{n} q_{n}-\Omega_{n}^{*} q_{n+1}\right)}\right], \quad \omega_{n}^{3}=\Omega_{n}^{3}=1 .
$$


3.4. Уравнения типа КдФ. Рассмотрим цепочки вида

$$
q_{n+1, x}=C\left(q_{n, x}, q_{n}, q_{n+1}\right), \quad C_{q_{n, x}} \neq 0 .
$$

Сама эта цепочка не может быть лагранжевой, но мы требуем этого от некоторого ее следствия. Пусть лагранжиан имеет вид (1), тогда уравнение Эйлера

$$
f q_{n+1, x}-f(-1) q_{n-1, x}+b_{0}+b_{1}(-1)=0, \quad f=-a_{1}
$$

должно обрашаться в тождество в силу (49). Кроме того, мы будем требовать отсутствия нетривиальных инвариантов (псевдоконстант):

$$
T(J)=J \Rightarrow J=\text { const }, \quad D(I)=0 \Rightarrow I=\text { const } .
$$

ПРЕДЛОЖЕНИЕ 3. Цепочки, удовлетворяющие даннбцм требованиям, имеют вид (с точностью до точечных замен)

$$
\begin{gathered}
q_{n+1, x}-\varepsilon q_{n, x}=G\left(q_{n+1}+\varepsilon q_{n}\right), \quad F=q_{n+1} q_{n, x}+\varepsilon b\left(q_{n+1}+\varepsilon q_{n}\right), \quad b^{\prime}=G \\
q_{n+1, x} q_{n, x}=\frac{1}{f\left(q_{n}, q_{n+1}\right)}, \quad F=q_{n, x} V\left(q_{n}, q_{n+1}\right), \quad f=V_{q_{n+1}} .
\end{gathered}
$$

ДокАЗАТЕЛЬСтво. Продифференцируем по $z=q_{x}$ тождество (50):

$$
\begin{aligned}
& f(1) C\left(C\left(z, q, q_{1}\right), q_{1}, q_{2}\right)-f z+b_{0}(1)+b_{1}=0 \Rightarrow \\
& \quad \Rightarrow f(1) C_{z}(1) C_{z}=f \Rightarrow C_{z z}(1) C_{z}^{2}+C_{z}(1) C_{z z}=0 .
\end{aligned}
$$

Комбинация этих соотношений дает $(T-1) C_{z z}^{2} /\left(f C_{z}^{3}\right)=0$, и в силу условия отсутствия инвариантов имеем $C_{z z}^{2}=\mathrm{const} \cdot f C_{z}^{3}$. Данное уравнение имеет два решения:

$$
C=A z+B, \quad C=\frac{k}{f z+A}+B
$$

где $A, B$, как и $f$, зависят от $q, q_{1}$, а $k$ - постоянная, которую можно считать равной единице за счет переопределения $f$.

В линейном случае имеем $f(1) A(1)=f / A=\rho^{2}\left(q_{1}\right)$, откуда легко получить $A=$ $\varepsilon \rho(q) / \rho\left(q_{1}\right), \varepsilon^{2}=1$. Точечная замена $q \rightarrow r(q)$ позволяет обратить $\rho$ в единицу. После этого тождество (53) дает $B(1)+b_{0}(1)=-\varepsilon B-b_{1}$ и приводит к цепочкам (51).

Для решений вида $C=1 /(f z+A)+B$ подстановка в (53) дает

$$
f(1) B+A(1)=0, \quad f(1) B(1)-f B(-1)+b_{0}+b_{1}(-1)=0 .
$$


Из первого равенства следует, что функция $B$ зависит только от $q_{1}$, причем цепочку (49) можно записать в виде

$$
\left(q_{n+1, x}-B\left(q_{n+1}\right)\right)\left(q_{n, x}-B\left(q_{n}\right)\right)=\frac{1}{f\left(q_{n}, q_{n+1}\right)} .
$$

Если $B=0$, то достаточно положить $b=0$ (случай $(52)$ ). Если $B \neq 0$, то замена $q \rightarrow$ $r(q)$ позволяет превратить $B$ в единицу, при этом

$$
f\left(q_{1}, q_{2}\right)-f\left(q_{0}, q_{1}\right)+b_{0}\left(q_{0}, q_{1}\right)+b_{1}(-1)\left(q_{-1}, q_{0}\right)=0,
$$

откуда получаем сначала $f_{1}=b_{1,0}=0$, но тогда и $f_{0}=0$. Таким образом, цепочка в данном случае тривиальна.

При помощи цепочек (51), (52) можно построить теорию ПБ для лагранжианов вида

$$
\mathcal{L}=\iint d x d t\left(\frac{1}{2} q_{t} q_{x}^{\alpha}+H\left(q, q_{x}, \ldots\right)\right), \quad \alpha= \pm 1,
$$

уравнение Эйлера для которых имеет вид

$$
J q_{t}=\frac{\delta H}{\delta q}, \quad J= \begin{cases}D_{x}, & \alpha=1 \\ q_{x}^{-2} D_{x}+D_{x} q_{x}^{-2}, & \alpha=-1\end{cases}
$$

В обоих случаях условие $\hat{L}-L \in \operatorname{Im} D_{t}+\operatorname{Im} D_{x}$ разбивается на два:

$$
\hat{q}_{t} \hat{q}_{x}^{\alpha}-q_{t} q_{x}^{\alpha} \in \operatorname{Im} D_{t}+\operatorname{Im} D_{x}, \quad \widehat{H}-H \in \operatorname{Im} D_{x} .
$$

При $\alpha=1$ первое выражение преобразуем следуюшим образом:

$$
\hat{q}_{t} \hat{q}_{x}-q_{t} q_{x}=\left(\hat{q}_{t}+\varepsilon q_{t}\right)\left(\hat{q}_{x}-\varepsilon q_{x}\right)+\varepsilon D_{t}\left(\hat{q} q_{x}\right)-\varepsilon D_{x}\left(\hat{q} q_{t}\right),
$$

что является полной дивергенцией, если $\hat{q}$ и $q$ связаны формулой (51). Аналогично в случае $\alpha=-1$ имеем

$$
\frac{\hat{q}_{t}}{\hat{q}_{x}}-\frac{q_{t}}{q_{x}}=D_{t}\left(V q_{x}\right)-D_{x}\left(V q_{t}\right),
$$

если $\hat{q}$ и $q$ связаны цепочкой вида (52). Для уточнения правой части цепочки используется условие $\widehat{H}-H \in \operatorname{Im} D_{x}$. 
Пример 6. Уравнение КдФ

$$
q_{t}=q_{x x x}-6 q_{x}^{2} \Rightarrow D_{x}\left(q_{t}\right)=\frac{\delta H}{\delta q}, \quad H=\frac{1}{2} q_{x x}^{2}+2 q_{x}^{3} .
$$

Ищем функцию $G$ такую, что $\widehat{H}-H$ есть полная производная в силу равенства $\hat{q}_{x}-$ $\varepsilon q_{x}=G(\hat{q}+\varepsilon q)$. Имеем

$$
\begin{gathered}
\hat{q}_{x x}^{2}-q_{x x}^{2}+4 \hat{q}_{x}^{3}-4 q_{x}^{3}=\left(\hat{q}_{x x}+\varepsilon q_{x x}\right)\left(\hat{q}_{x}+\varepsilon q_{x}\right) G^{\prime}+4 \hat{q}_{x}^{3}-4 q_{x}^{3}= \\
=\frac{1}{2} D_{x}\left(\left(\hat{q}_{x}+\varepsilon q_{x}\right)^{2} G^{\prime}\right)-\frac{1}{2}\left(\hat{q}_{x}+\varepsilon q_{x}\right)^{3} G^{\prime \prime}+4 \hat{q}_{x}^{3}-4 q_{x}^{3} \stackrel{\operatorname{Im} D_{x}}{=} \\
\stackrel{\operatorname{Im} D_{x}}{=} 4\left(\varepsilon q_{x}+G\right)^{3}-4 q_{x}^{3}-\frac{1}{2}\left(2 \varepsilon q_{x}+G\right)^{3} G^{\prime \prime} .
\end{gathered}
$$

Коэффициент при $q_{x}^{3}$ равен $\varepsilon-1-\varepsilon G^{\prime \prime}$, откуда получаем $G^{\prime \prime}=1-\varepsilon$. Случай $\varepsilon=1$ неинтересен, так как приводит к $\hat{q}_{x}=q_{x}$. Продолжая выгисление при $\varepsilon=-1$, получаем окончательно ПБ в виде

$$
\hat{q}_{x}+q_{x}=(\hat{q}-q)^{2}+\beta .
$$

Параметр $\beta$ можно выбирать разным на каждом шаге. Обозначая $f=\hat{q}-q$, получим одевающую цепочку (8).

ПримеР 7. Уравнение sin-Гордон

$$
q_{x t}=\sin 2 q, \quad H=\frac{1}{2} \cos 2 q .
$$

В данном случае $\varepsilon$ принимает два значения $\varepsilon= \pm 1$, так как разность

$$
\cos 2 \hat{q}-\cos 2 q=-2 \sin (\hat{q}-q) \sin (\hat{q}+q)
$$

есть полная производная при $\hat{q}_{x}-\varepsilon q_{x}=\beta \sin (\hat{q}+\varepsilon q)$.

Обсудим кратко связь между одевающими цепочками для этих двух классических примеров. Переходя к уравнению sh-Гордон $q_{x y}+e^{2 q}-e^{-2 q}=0$ и используя оба знака, находим

$$
\begin{aligned}
\gamma_{j}^{-1} \partial_{x}\left(q_{j}+q_{j+1}\right) & =e^{q_{j+1}-q_{j}}-e^{q_{j}-q_{j+1}}, \\
\gamma_{j} \partial_{y}\left(q_{j}-q_{j+1}\right) & =e^{q_{j+1}+q_{j}}-e^{-q_{j}-q_{j+1}} .
\end{aligned}
$$

Легко проверить, что условие антипериодичности

$$
q_{j+l}=-q_{j} \quad \forall j, \quad l=2,4, \ldots,
$$

сводит обе одевающие цепочки (57), (58) к хорошо определенным динамическим системам и что соответствующие векторные поля $D_{x}, D_{y}$ коммутируют. Таким образом, система уравнений $(57),(58)$ и (59) описывает достаточно богатый класс частных решений уравнения $q_{x y}+e^{2 q}-e^{-2 q}=0$. С другой стороны, можно проверить, что условие (59) и формулы

$$
f_{j}=q_{j, x}-\gamma_{j} e^{q_{j+1}-q_{j}} \quad(j=1,2, \ldots, l), \quad f_{l+1}=-q_{1, x}
$$

приводят нас к $(l+1)$-периодическому замыканию одеваюшей цепочки $(8)$, рассмотренному в работе [2]. 
ПримеР 8. Лагранжиан для уравнения Кричевера-Новикова

$$
q_{t}=q_{x x x}-\frac{3}{2 q_{x}}\left(q_{x x}^{2}-r(q)\right)+c q_{x}
$$

имеет плотность

$$
L=\frac{q_{t}}{q_{x}}+\frac{r(q)-q_{x x}^{2}}{q_{x}^{2}}, \quad r^{(\mathrm{V})}(q)=0,
$$

т.е. в данном случае ПБ следует искать в виде (52). Ответ имеет вид [14]

$$
q_{x} \hat{q}_{x}=h(q, \hat{q})=\left(f_{\hat{q}}(q, \hat{q})\right)^{-1}
$$

где $h(q, \hat{q})$ - полином второй степени по $q$ и $\hat{q}$ такой, что $r(q)=2 h h_{\hat{q} \hat{q}}-h_{\hat{q}}^{2}$. Лагранжиан цепочки (50) легко определяется:

$$
L=\sum_{n} f\left(q_{n-1}, q_{n}\right) q_{n, x}, \quad f(q, \hat{q})=\frac{2}{\sqrt{r(q)}} \operatorname{arctg} \frac{h_{\hat{q}}(q, \hat{q})}{\sqrt{r(q)}} .
$$

3.5. Уравнения типа Савады-Котера. В общем случае $L=D^{3}+u D+v$ уравнение Лакса

$$
\begin{gathered}
L_{t}=[A, L], \quad A=D^{5}+a D^{3}+b D^{2}+c D+\hat{c}, \\
3 a=5 u, \quad 3 b=5\left(u_{x}+v\right), \quad 9 c=10 u_{x x}+5 u^{2}+15 v_{x}, \quad 9 \hat{c}=10\left(v_{x x}+u v\right),
\end{gathered}
$$

приводит к следуюшей паре уравнений для коэффициентов оператора $L$ :

$$
\begin{array}{r}
9 u_{t}+u_{x x x x x}+5 D\left(u u_{x x}+3 u_{x} v+\frac{1}{3} u^{3}-3 v^{2}\right)=0, \\
9 v_{t}+v_{x x x x x}+5 D\left(u v_{x x}+2 u_{x x} v+2 u_{x} v_{x}-3 v v_{x}+u^{2} v\right)=0 .
\end{array}
$$

Легко проверить, что эта система допускает редукции

$$
v=0, \quad v=u_{x}, \quad 2 v=u_{x}
$$

сводящие ее к скалярному эволюционному уравнению пятого порядка. Все эти редукции сводятся простыми дифференциальными подстановками (см. ниже) к одному и тому же уравнению

$$
9 f_{t}+D\left(f_{x x x x}+f^{5}\right)=5 D\left(f_{x x} f_{x}+f^{2} f_{x x}+f f_{x}^{2}\right) .
$$

Здесь $f=(\ln \psi)_{x}$, и в нередуцированном виде это уравнение совпадает с уравнением $\psi_{t}=A \psi \Leftrightarrow 9 \psi_{t}=\left(6 v-3 u_{x}+9 \lambda\right) \psi_{x x}+\left(u_{x x}-u^{2}-3 v_{x}\right) \psi_{x}+\left(v_{x x}+4 u v+6 \lambda u\right) \psi$ 
Заметим, что в случае уравнения Буссинеска

$$
L_{y}=\left[D^{2}+a, L\right] \Rightarrow \phi_{y y}+\frac{1}{3} \phi_{x x x x}+2 \phi_{x x} \phi_{y}=2 \phi_{x}^{2} \phi_{x x}, \quad \phi=\ln \psi .
$$

Последнее уравнение для $\phi=\ln \psi$ естественно рассматривать как модифицированное уравнение Буссинеска. В рассматриваемом случае, т.е. для оператора $A$ пятого порядка, в качестве дополнительной к $\phi=\ln \psi$ динамической переменной можно выбрать $u$. Исключив в уравнении $\psi_{t}=A \psi$ функцию $v$ при помоши уравнения (ср. (9))

$$
f_{x x}+3 f f_{x}+f^{3}+u f+v=\lambda, \quad f=\phi_{x},
$$

получаем одно из уравнений системы

$9 \phi_{t}+f_{x x x x}+6 f^{5}+15\left(f_{x} f_{x x}+f f_{x}^{2}+f^{3} f_{x}\right)+5\left(u f_{x}\right)_{x}+15 u f f_{x}+10 u f^{3}+5 u^{2} f=15 \lambda g$,

где

$$
f=\phi_{x}, \quad g=\phi_{y}=f_{x}+f^{2}+\frac{2}{3} u .
$$

В качестве второго уравнения модифишированной системы может служить уравнение (64) после исключения $v$ при помощи (68).

Рассмотрим редукции цепочки (10) с дополнительными условиями

$$
q_{3 n}=q_{3 n+l}, \quad l=1,2,
$$

связанные, как будет показано ниже, с условиями (66). Напомним, что в переменных $f$, $u=3 z$ уравнения цепочки записываются следуюшим образом:

$$
\left(z_{j-1}+z_{j}+z_{j+1}\right)_{x}-3 f_{j-1} z_{j-1}+3 f_{j} z_{j+1}-f_{j-1}^{3}+f_{j}^{3}=\alpha_{j} .
$$

При изучении указанных редукций, как мы увидим, полезно использовать также пару переменных $f, g$, указанных в (70). Точнее, в силу уравнений цепочки (10) выполняются соотношения

$$
\begin{gathered}
g_{j}=f_{j}^{2}+z_{j}+z_{j+1}, \quad f_{j, x}=z_{j+1}-z_{j} \Rightarrow v_{j+1}=v_{j}+\frac{3}{2} D\left(g_{j}+f_{j, x}\right), \\
u_{j+1}=u_{j}+3 D f_{j},
\end{gathered}
$$

указывающие связь переменных $f, g$ с законами сохранения цепочки. Отметим, наконец, что в силу определений (72) при всех $j$ должны выполняться уравнения

$$
\left(f_{j}+f_{j+1}\right)_{x}=f_{j}^{2}-f_{j+1}^{2}+g_{j+1}-g_{j} .
$$

При $l=1$ и $f_{3 n}=\mu_{3 n}=0$ мы имеем $v_{3 n}=0$ в силу (68) и уравнения цепочки (71) сводятся к системе трех уравнений для $f_{1}, f_{2}, g, \hat{g}$ :

$$
g_{x}+f_{1} g=\alpha_{1}, \quad \hat{g}_{x}-f_{2} \hat{g}=\alpha_{3}, \quad g+\hat{g}=\gamma,
$$


где $f_{k}=f_{3 n+k}$ и

$g=g_{1}+z_{1}=f_{1}^{2}+2 z_{1}+z_{2}, \quad \hat{g}=g_{2}+z_{3}=f_{2}^{2}+2 z_{3}+z_{2}, \quad \gamma_{x}=\alpha_{1}+\alpha_{2}+\alpha_{3}=0$.

Из уравнений (74), (73) следует, что

$$
\begin{gathered}
\gamma f_{1}=f \hat{g}+\alpha, \quad \gamma f_{2}=f g-\alpha, \quad f=f_{1}+f_{2}, \quad \alpha=\alpha_{1}+\alpha_{3}, \\
2 f_{x}=f\left(f_{1}-f_{2}\right)+\hat{g}-g, \quad \hat{g}-g=2 \frac{\gamma f_{x}-\alpha f}{f^{2}+\gamma} .
\end{gathered}
$$

При $|\alpha|+|\gamma| \neq 0$ решение цепочки вырождается.

При $\alpha=\gamma=0$ находим, что $\hat{g}=g=0$,

$$
2\left(f_{1}+f_{2}\right)_{x}=f_{1}^{2}-f_{2}^{2}, \quad v_{0}=v_{3}=0, \quad v_{1}=u_{1, x}, \quad v_{2}=\frac{1}{2} u_{2, x} .
$$

При этом с помощью соотношений

$$
u_{2}=3 z_{2}=-2 f_{2, x}-f_{2}^{2}, \quad u_{3}=3 z_{3}=f_{2, x}-f_{2}^{2}
$$

и подстановки $u=-2 f_{x}-f^{2}$ в уравнение (69) получаем (67). Несложно проверить также, что эта дифференциальная подстановка переводит решения уравнения (67) в решения уравнения Каупа-Купершмидта

$$
u_{t}=u_{x x x x x}+5 D\left(u u_{x x}+\frac{3}{4} u_{x}^{2}+\frac{1}{3} u^{3}\right)
$$

соответствуюшего редукции $v=u_{x} / 2$ в системе уравнений (64), (65). Аналогично вторая дифференциальная подстановка $u=f_{x}-f^{2}$ переводит решения уравнения (67) в решения уравнения Савады-Котера

$$
u_{t}=u_{x x x x x}+5\left(u u_{x x}\right)_{x}+5 u^{2} u_{x}
$$

соответствуюшего редукции $v=0$ в системе уравнений (64), (65). Оставшаяся редукция $v=u_{x}$ соответствует $j=1$. При этом

$$
u_{1}=3 z_{1}=-f_{1, x}-f_{1}^{2}
$$

и функция $f=-f_{1}$ является решением уравнения (67).

Описанный выше набор дифференциальных подстановок эквивалентен преобразованию Дарбу

$$
L=D^{3}+\frac{1}{2} D u+\frac{1}{2} u D=(D+f) D(D-f) \rightarrow \hat{L}=(D-f)(D+f) D=D^{3}+\hat{u} D,
$$


где $u$ - решение уравнения Савады-Котера, а $\hat{u}$ - решение уравнения Каупа-Купершмидта.

В случае $l=2$ при $f_{3 n}+f_{3 n+1}=\mu_{3 n}+\mu_{3 n+1}=0$ находим, что

$$
v_{1}=\frac{3}{2} z_{1, x}, \quad v_{4}=\frac{3}{2} z_{4, x},
$$

и четыре уравнения с $j=3 n, \ldots, 3 n+3$ образуют замкнутую систему уравнений

$$
\begin{aligned}
f_{1, x x}+3 f_{1} z_{2}+f_{1}^{3}+\frac{3}{2} z_{1, x} & =\mu_{1}, \\
f_{2, x x}+3 f_{2} z_{3}+f_{2}^{3}-3 f_{1} g_{1}-3 f_{1} z_{2} & =\mu_{2}-3 \mu_{1}, \\
f_{3, x x}+3 f_{3} z_{3}+f_{3}^{3}-\frac{3}{2} z_{4, x} & =\mu_{3}
\end{aligned}
$$

вместе с первым интегралом

$$
g_{1}+g_{2}+g_{3}=\gamma
$$

Отметим, что первое и третье из уравнений этой системы можно переписать в виде

$$
g_{x}+2 f_{1} g=2 \mu_{1}, \quad \hat{g}_{x}-2 f_{3} \hat{g}=-2 \mu_{3}, \quad g=g_{1}+z_{2}, \quad \hat{g}=g_{3}+z_{3},
$$

а оставшееся второе уравнение дает

$$
f_{x x}+\frac{3}{2}\left(z_{1}+z_{4}\right) f=\frac{1}{2} f^{3}-\frac{3}{2} f^{2}\left(f_{1}+f_{3}\right)+3 f_{1} f_{3}+\frac{3}{2} f_{x}\left(f_{1}-f_{3}\right)+\frac{3}{2} \gamma f_{2}+\alpha_{3}-\alpha_{2} .
$$

После преобразований это уравнение принимает следующий вид:

$$
\begin{aligned}
& f_{x x}+\frac{3}{2}\left(z_{1}+z_{4}\right) f+\frac{1}{4} f^{3}+\frac{3}{4} \frac{\gamma^{2}-f_{x}^{2}}{f}= \\
&= \frac{3}{4} f\left[\left(f_{2}+\frac{\gamma}{f}\right)^{2}-\left(f_{3}-f_{1}+\frac{f_{x}}{f}\right)^{2}\right]+\alpha_{3}-\alpha_{2} . \\
& 2 v_{1}=3 z_{1, x}, \quad 2 v_{4}=3 z_{4, x}, \quad g+\hat{g}+f_{2}^{2}=\gamma
\end{aligned}
$$

В работе [15] показано, что ПБ имеет вид

$2\left(q_{1}-q\right)_{x x}\left(q_{1}-q\right)+\left(q_{1}-q\right)^{2}\left(q_{1, x}+q_{x}\right)-\frac{3}{2}\left(q_{1, x}-q_{x}\right)^{2}+\frac{1}{18}\left(q_{1}-q\right)^{4}+\mu\left(q_{1}-q\right)=0$.

При $\mu=0$ оно найдено в работе [16] (см. также [17], [18]). Это ПБ соответствует, очевидно, обрашению в нуль квадратной скобки в правой части (79), следуюшему из условия совместности.

Благодарности. Работа поддержана грантами РФФИ № 99-01-00431 и INTAS № 99-01782. 


\section{Список литературы}

[1] А. Б. ШШабат. ТМФ. 1999. Т. 121. № 1. С. 165-176.

[2] А. П. Веселов, А. Б. Шабат. Функц. анализ и его прилож. 1993. Т. 27. № 2. С. 1-21.

[3] J. Weiss. J. Math. Phys. 1987. V. 28. № 9. P. 2025-2039.

[4] Xing-Biao Hu, Hon-Wah Tam. Inverse Problems. 2001. V. 17. P. 319-327.

[5] A.K. Svinin. Inverse Problems. 2001. V. 17. P. 307-318.

[6] О. И. Богоявленский. Опрокидывающиеся солитоны. Нелинейные интегрируемые уравнения. М.: Наука, 1991.

[7] А. В. Михайлов, А. Б. Шабат, Р. И. Ямилов. УМН. 1987. Т. 42. № 4. С. 3-53.

[8] В. Э. Адлер, А. Б. Шабат. ТМФ. 1997. Т. 112. № 2. С. 179-194.

[9] В. Г. Марихин. Письма в ЖЭТФ. 1997. Т. 66. С. 673-678.

[10] А. Б. Шабат, Р. И. Ямилов. Алгебра и анализ. 1990. Т. 2. № 2. С. 183-208.

[11] R. I. Yamilov. Classification of Toda type scalar lattices. In: Proc. NEEDS'92. Eds. V. Makhankov, I. Puzynin, and O. Pashaev. Singapore: World Scientific, 1993. P. 423-431.

[12] В. Э. Адлер. ТМФ. 2000. Т. 124. № 1. C. 48-61.

[13] E. К. Склянин. Функц. анализ и его прилож. 1982. Т. 16. № 4. С. 27-34.

[14] V. E. Adler. Int. Math. Research Notices. 1998. № 1. P. 1-4.

[15] R. Conte. Exact solutions of nonlinear partial differential equations by singularity analysis. In: Direct and inverse methods in nonlinear evolution equations. CIME school (Cetraro, 5-12 September 1999). Ed. A. Greco. Berlin: Springer, 2000; nlin.SI/0009024.

[16] C. Rogers, S. Carillo. Physica Scripta. 1987. V. 36. P. 865-869.

[17] M. C. Nucci. J. Phys. A. 1989. V. 22. P. 2897-2913.

[18] J. Satsuma, D. J. Kaup. J. Phys. Soc. Japan. 1977. V. 43. P. 692-697. 